\title{
Clinical Characteristics and Follow-Up Data in a Series of Twenty Infective Endocarditis Complicated of Rheumatic Manifestations
}

\author{
Madiha Mahfoudhi1,2*, Imen Gorsane1,2, Hedia Bellali ${ }^{3}$, Amel Gaieb Battikh ${ }^{1,2}$, \\ Sami Turki1,2, Fathi Ben Hamida ${ }^{1,2}$, Taïeb Ben Abdallah ${ }^{1,2}$ \\ ${ }^{1}$ Internal Medicine A Department, Charles Nicolle Hospital, Tunis, Tunisia \\ ${ }^{2}$ Research Unit of Kidney Diseases (LROOSP01), Charles Nicolle Hospital, Tunis, Tunisia \\ ${ }^{3}$ Epidemiology and Statistics Department, A Mami Hospital, Ariana, Tunisia \\ Email: madiha mahfoudhi@yahoo.fr
}

Received 15 March 2015; accepted 26 May 2015; published 29 May 2015

Copyright (C) 2015 by authors and Scientific Research Publishing Inc.

This work is licensed under the Creative Commons Attribution International License (CC BY).

http://creativecommons.org/licenses/by/4.0/

(c) (i) Open Access

\begin{abstract}
The infective endocarditis is a septicemia complicated of many systemic manifestations. Rheumatic manifestations can be revelatory, leading sometimes to a lateness diagnosis. The aim of this study is to determine the epidemiological, clinical, bacteriological profile and the follow up in patients affected of an infective endocarditis complicated of rheumatic manifestations. It's a retrospective study concerning the period from January 1990 to December 2015. The analysis had implicated epidemiological, clinical, biological data, in association to immunological, bacteriological and radiological examinations. All the patients fulfilled the revised Duke's criteria for the infective endocarditis. Articular and osseous radiographs, blood culture, transthoracic and/or transoesophageal echocardiography were performed in all patients. Twenty patients had rheumatic manifestations. The mean age was 37 years. There were arthralgia in 15 cases, myalgia in 5 cases and arthritis in 8 cases: 6 cases of mono-arthritis and 2 cases of oligo-arthritis. All patients had an inflammatory biological syndrome. The blood culture was positive in all the cases. Echocardiography revealed vegetations in all the patients: mitral in 10 cases, aortic in 4 cases, mitral and aortic in 4 cases, aortic and tricuspid in 1 case, tricuspid in 1 case. The radiological examination found 2 cases of spondylodiscitis and a case of saco-iliitis. The articular outcome was favorable in 17 cases. Three patients had died. The infective endocarditis should be considered in case of febrile arthritis. An early diagnosis and adapted treatment are guarantors of better prognosis.
\end{abstract}

\section{Keywords}

Infective Endocarditis, Blood Culture, Vegetation, Myalgia, Arthritis

\footnotetext{
${ }^{*}$ Corresponding author.
} 


\section{Introduction}

Revelatory manifestations of the infective endocarditis (IE) are varied, causing a diagnostic difficulty. Rheumatic involvement is common in the EI. Lateness in diagnosis may occur if the articular manifestations are at the forefront of the clinical feature, especially if there is a heart murmur lack [1]-[4]. These manifestations are most often arthralgia. Arthritis is less frequent corresponding to either a septic location which is exceptional or immune dysfunction. Articular prognosis depends on diagnosis delay and efficiency of treatment.

\section{Materials and Methods}

This is a cross-sectional study of 24 years (1990-2015) that has interested 20 patients admitted to the internal medicine A in Charles Nicolle Hospital for an IE and presenting different rheumatic manifestations. The analysis concerned the gender, age, functional and physical signs of patients, laboratory tests, immunological, bacteriological and radiological results and the follow up of all patients. The point of entrance has been searched in all cases. Blood culture and transthoracic and/or transoesophageal echocardiography were performed in all cases. None of our patients benefited from cardiac or synovial biopsy.

The diagnosis was based on the presence of two major criteria of Duke, modified by Li et al. [5]. All patients were treated with antibiotics.

\section{Results}

Of 45 patients affected of IE, 20 patients (44.44\%) had a rheumatic disorder. The sex ratio was 15/5 (3). Their average age was 37 years (ranging 21 to 61 years). In the past medical history, two patients were followed for mitral valvular stenosis, a patient was a toxicoman, and two others had a history of rheumatic fever.

Fever was objectified in the 20 cases, while the heart murmur was found in 15 cases.

Arthralgia was reported in 15 cases implicating Knees in 7 cases, knees and elbows in 5 cases and low back in 3 cases. Myalgia was found in 5 cases and arthritis in 8 cases: 6 cases of mono-arthritis and 2 cases of oligoarthritis.

Joint involvement was revelatory of IE in $45 \%$ of cases.

Three men aged respectively of 25, 39 and 45 years had arthritis of knee.

Arthritis of the left ankle was diagnosed in a 23 years old man. Another 43 years old man presented left sacroiliitis. In addition, arthritis of the proximal interphalangeal joint of the first right finger complicated an IE feature in a 34 years old woman. Arthritis of knees and ankle were shown in 2 women.

The radiological diagnosis showed 2 cases of spondylodiscitis touching the 4th dorsal vertebra and one case of left sacroiliitis.

Vascular purpura of legs was found in 3 patients; its pathological examination confirmed the presence of leukocytoclastic vasculitis.

Neurological and ophthalmologic examination showed no abnormalities.

Regarding biological investigations, renal and liver functions were normal. Proteinuria per 24 hours was negative. There was no microscopic hematuria.

All patients had inflammatory syndrome with a C-reactive protein average level of $90 \mathrm{mg} / \mathrm{L}$. Normocytic normochromic inflammatory anemia was found in 16 cases with an average hemoglobin level of $10.5 \mathrm{~g} / \mathrm{dl}$. Neutrophilic leukocytosis was revealed in all the cases. In addition, 10 patients had thrombocytosis. The muscle enzyme levels were normal in all cases.

Immunologically, the direct Coombs test was positive in 14 patients. Anti-nuclear antibodies, antiphospholipid antibodies, and anti-CCP antibodies were negative. Rheumatoid factor was positive in 10 cases. Circulating immune complexes (CIC) were present in 8 cases. The dosage of C3 and/or C4 was low in 8 patients.

Articular punction performed in 2 cases for knee arthritis isolated no germ.

No synovial biopsy was performed.

Blood cultures were positive in all the cases.

Isolated germs were:

Staphylococcus aureus (6 cases)

Streptococcus viridans (9 cases)

Enterobacter (4 cases) 
Corynobacterium (1 case)

The point of entrance was skin in 4 cases, oto-rhino-laryngology system in 7 cases, urinary tract in 3 cases, digestive system in one case and not found in 5 patients.

The valve disease was objectified by transthoracic echocardiography in 12 cases and transoesophageal echocardiography in 8 cases. Mitral localization was diagnosed in 10 cases, aortic in 4 cases, mitral and aortic in 4 cases, aortic and tricuspid in one case, tricuspid in one case.

All patients received medical treatment based on adapted antibiotics.

The articular outcome was good in 17 patients, the other three patients died. One of them aged of 34 years old had died in an acute lung edema complicating his aortic and mitral valve damage.

The other was a 36 year old drug addict who had died in a septic shock.

The third was a 40 year old patient who died because of a fatal and rapid valvular and heart strings damage.

The articular and cardiac outcome was favorable in 17 patients. It was marked by the apyrexia, disappearance of rheumatic disturbances and inflammatory syndrome and negativation of blood cultures.

None of these patients had received an anti-inflammatory. Articular prognosis was good; they had no articular limitation or deformation.

\section{Discussion}

The IE is a valve or a cardiac prosthesis graft of a microorganism. The clinical feature is polymorphic manifesting essentially by a prolonged fever and a heart murmur present in almost $95 \%$ of cases. Various systemic manifestations (skinny, renal, neurological, rheumatologic signs) can complicate the IE [1].

Rheumatic manifestations are frequent in the IE, but they are not specific to the disease and can accompany many other diseases. They can delay diagnosis especially in the absence of a heart murmurs; thus worsening the functional and vital prognosis of this severe systemic disease [2].

Pathogeny of IE involves either hematogenous septic emboli migration causing septic arthritis, or more frequently production of auto-antibodies responsible of tissue damage in multiple joints [2].

The prevalence of immunological abnormalities varies according to published series. There is a correlation between the presence of CIC and the occurrence of extra-cardiac localizations [3].

Several immunological abnormalities may accompany EI: presence of rheumatoid factor, hypocomplementemia, presence of CIC, cryoglobulinemia , antinuclear antibodies and anticardiolipin antibodies [2] [3].

Thus, in our patients presenting IE with rhumathological complications, the CIC were present in $40 \%$ of cases, rheumatoid factor was found in $50 \%$ of our patients.

The diagnosis of IE should be considered in case of unexplained fever associated to rheumatic plaints. Cardiac auscultation reveals a heart murmur. Achieving repeated sets of blood cultures in aerobic and anaerobic environments is essential even if blood culture is negative.

Practice of transthoracic and/or transoesophageal echocardiography is indicated to search for a valvular thickening or vegetations and valvular damage, or cardiac abscess and to judge about size and mobility of vegetations.

The diagnosis of IE was considered certain in all patients in our series because of the presence of two major criteria for diagnostic classification of infective endocarditis according to the Duke criteria, modified by Li et al [4].

The combination of polymorphic rheumatologic manifestations with fever (100\% of cases in our series), inflammatory syndrome (100\% of our patients), and immunological abnormalities such as the presence of rheumatoid factor ( $50 \%$ in our series) can be confused with other pathologies such as connective diseases and neoplasia. Hence, the contribution of the revised classification of Duke in the diagnosis of IE in cases of atypical clinical presentation is important.

None of our patients had benefited of a cardiac histological examination since they were not candidates for surgery.

Musculoskeletal manifestations of IE associated arthralgia including low back pain, and inflammatory or septic arthritis, spondylodiscitis, sacroiliitis, osteitis, myalgia, tenosynovitis and bursitis [5] [6].

The rheumatic disorder is often indicative and may delay diagnosis when it is isolated in the beginning.

Arthralgia is the most frequent, being reported in $50 \%$ of cases of IE. It mainly affects the shoulders, knees, hips, ankles. There is mono-or oligo-arthritis in half of the cases [5] [6]. 
Peripheral arthritis is seen in $20 \%$ - $50 \%$ of patients with rheumatologic events corresponding to a mono or oligo-arthritis interesting shoulder, knee or ankle [5] [6].

Reactive arthritis is mostly found, septic arthritis is rare. Progress in bacteriological research has enabled new studies to publish more cases of septic arthritis occurring in the course of an IE [7] [8].

In our series, no case of septic arthritis was reported.

Reactive arthritis was observed in $40 \%$ of our patients; whereas arthralgia presented $75 \%$ of all cases.

Articular punction must be made to eliminate septic arthritis that may engage the joint outcome [7] [8]. The result is usually an inflammatory aspect of the articular liquid. Some authors recommend performing a synovial biopsy.

Radiological results have no specificity, whether by performing joint and bone radiographs or CT and MRI images [7]-[9].

There is no correlation between joint damage and the affected valve [8] [10]-[12].

Some studies reported no significant difference in the distribution of isolated germ according to the presence or absence of rheumatic manifestations. Others noted the predominance of certain bacteria in patients with a rheumatic disorder such as group D Streptococcus [6], Streptococcus bovis [13] and Enterococcus [14].

In our series, there was predominance of Streptococcus Viridans (45\% of cases), Staphylococcus Aureus (30\% of cases) and Enterobacter (20\% of cases).

Treatment is based on prolonged antibiotic therapy adapted to the responsible germ, and surgical treatment in certain indications. The IE prognosis is determined by the severity of cardiac complications. The administration of efficient antibiotic treatment is marked by the disappearance of rheumatic manifestations, infectious and biological inflammatory syndrome and immunological abnormalities [15].

\section{Conclusion}

The diagnosis of IE should be considered in case of arthralgia or febrile arthritis. The early diagnosis and urgent treatment improve the IE prognosis.

\section{Disclosure Statement}

The authors have nothing to disclose.

\section{References}

[1] Moreillon, P. and Que, Y.A. (2004) Infective Endocarditis. The Lancet, 363, 139-149. http://dx.doi.org/10.1016/S0140-6736(03)15266-X

[2] Beynon, R.P., Bahl, V.K. and Prendergast, B.D. (2006) Infective Endocarditis. BMJ, 333, 334-339. http://dx.doi.org/10.1136/bmj.333.7563.334

[3] Bayer, A.S., Theofilopoulos, A.N., Eisenberg, R., Dixon, F.J. and Guze, L.B. (1976) Circulating Immune Complexes in Infective Endocarditis. The New England Journal of Medicine, 295, 1500-1505. http://dx.doi.org/10.1056/NEJM197612302952703

[4] Li, J.S., Sexton, D.J., Mick, N., Nettles, R., Fowler Jr., V.G., Ryan, T., et al. (2000) Proposed Modifications to the Duke Criteria for the Diagnosis of Infective Endocarditis. Clinical Infectious Diseases, 30, 633-638. http://dx.doi.org/10.1086/313753

[5] Sapico, F.L., Liquete, J.A. and Sarma, R.J. (1996) Bone and Joint Infection in Patients with Infective Endocarditis: Review of a 4-Year Experience. Clinical Infectious Diseases, 22, 783-787. http://dx.doi.org/10.1093/clinids/22.5.783

[6] Thomas, P., Allal, J., Bontoux, D., Rossi, F., Poupet, J.Y., Petitalot, J.P., et al. (1984) Rheumatological Manifestations of Infective Endocarditis. Annals of the Rheumatic Diseases, 43, 716-720. http://dx.doi.org/10.1136/ard.43.5.716

[7] Roberts-Thomson, P.J., Rischmueller, M., Kwiatek, R.A., Soden, M., Ahern, M.J., Hill, W.R., et al. (1992) Rheumatic Manifestations of Infective Endocarditis. Rheumatology International, 12, 61-63. http://dx.doi.org/10.1007/BF00300978

[8] Gonzales-Juanatey, C., Gonzalez-Gay, M.A., Llorca, J., Crespo, F., García-Porrúa, C., Corredoira, J., et al. (2001) Rheumatic Manifestations of Infective Endocarditis in Non-Addicts. A 12-Year Study. Medicine, 80, 9-19. http://dx.doi.org/10.1097/00005792-200101000-00002

[9] Churchill, M.A., Geraci, H.J.E. and Hunder, G.G. (1977) Musculoskeletal Manifestations of Bacterial Endocarditis. Annals of Internal Medicine, 87, 754-759. http://dx.doi.org/10.7326/0003-4819-87-6-754 
[10] Meyers, O.L. and Commerford, P.J. (1977) Musculoskeletal Manifestations of Bacterial Endocarditis. Annals of Rheumatic Diseases, 36, 517-519. http://dx.doi.org/10.1136/ard.36.6.517

[11] Levo, Y. and Nashif, M. (1983) Musculoskeletal Manifestations of Bacterial Endocarditis. Clinical and Experimental Rheumatology, 1, 49-52.

[12] Wofsy, D. (1980) Culture-Negative Septic Arthritis and Bacterial Endocarditis. Diagnosis by Synovial Biopsy. Arthritis \& Rheumatism, 23, 605-607. http://dx.doi.org/10.1002/art.1780230513

[13] Roberts-Thomson, P.J., Rischmueller, M., Kwiatek, R.A., Soden, M., Ahern, M.J., Hill, W.R., et al. (1992) Rheumatic Manifestations of Infective Endocarditis. Rheumatology International, 12, 61-63. http://dx.doi.org/10.1007/BF00300978

[14] Vlahakis, N.E., Temesgen, Z., Berbari, E.F. and Steckelberg, J.M. (2003) Osteoarticular Infection Complicating Enterococcal Endocarditis. Mayo Clinic Proceedings, 78, 623-628. http://dx.doi.org/10.4065/78.5.623

[15] Cabell, C.H., Jollis, J.G., Peterson, G.E., Corey, G.R., Anderson, D.J., Sexton, D.J., et al. (2002) Changing Patient Characteristics and the Effect on Mortality in Endocarditis. Archives of Internal Medicine, 162. 90-94.

http://dx.doi.org/10.1001/archinte.162.1.90 